\title{
CENTROS PARAMAGNÉTICOS EM ELBAÍTA ROSA NATURAL E IRRADIADA
}

\author{
Cristiane Castañeda ${ }^{1}$, Nilson Francisquini Botelho ${ }^{2}$, Klaus Krambrock ${ }^{3}$, \\ Maria Sylvia Dantas ${ }^{3} \&$ Antônio Carlos Pedrosa-Soares ${ }^{1}$
}

\begin{abstract}
Spectroscopy study of natural and treated pink tourmalines from Minas Gerais, Brazil, using electron paramagnetic resonance (EPR), optical detection of electron paramagnetic resonance (ODEPR), optical absorption and Raman spectroscopy is reported. Electron-microprobe analyses indicate that all samples are close elbaite end-member. EPR spectra of natural pink elbaite indicate the presence of $\mathrm{Mn}^{2+}$ in to distinct $\mathrm{Y}$ site. Optical absorption measurements show three dominant absorption bands centered at 460,520 and $680 \mathrm{~nm}$ in the pink tourmalines, which can be related with optical transitions of $\mathrm{Mn}^{2+}$. Different defects have been identified by EPR like $\mathrm{Mn}^{2+}, \mathrm{Fe}^{3+}, \mathrm{H}^{0}$ and $\mathrm{O}^{-}$produced by g-irradiation in pale pink elbaite. The last one is a hole trap of $\mathrm{O}$ and considered a color center. It shows mainly superhyperfine interaction with two $\mathrm{Al}$ nuclei. Atomic hydrogen $\mathrm{H}^{0}$ is as electron trap whereas the $\mathrm{Fe}^{3+}$ is an electron precursor. From irradiation and heat treatments we conclude that the $\mathrm{Mn}^{2+}$ is the precursor of the pink color in elbaites. Irradiation intensifies the pink color whereas heat treatments at about $450^{\circ} \mathrm{C}$ decolorize the pink tourmaline.
\end{abstract}

Keywords: tourmalines, EPR, gama irradiation.

\section{RESUMO}

Estudos espectroscópicos em turmalinas rosas naturais e tratadas de Minas Gerais, Brasil, foram feitos usando ressonância paramagnética eletrônica (EPR), detecção óptica da ressonância magnética (ODMR), absorção óptica e espectroscopia Raman. Microanálises indicam que todas as amostras têm composições próximas às do membro final elbaíta. O espectro EPR indica a presença de $\mathrm{Mn}^{2+}$. Medidas de absorção óptica mostram três bandas de absorção dominantes centradas a 460, 520 e 680 $\mathrm{nm}$, atribuídas às transições ópticas do $\mathrm{Mn}^{2+}$. Diferentes defeitos foram identificados na elbaíta rosa por EPR, como $\mathrm{Mn}^{2+}, \mathrm{Fe}^{3+}, \mathrm{H}^{0}$ e $\mathrm{O}^{-}$produzidos por g-irradiação. $\mathrm{O}$ último é um centro do tipo buraco do tipo $\mathrm{O}^{-}$e mostra interação superhiperfina com dois Al. É considerado como um centro de cor amarelo do tipo Al-O-Al. O átomo de hidrogênio $\mathrm{H}^{0}$ é um centro de aprisionamento de elétrons, ao passo que o $\mathrm{Fe}^{3+}$ é um precursor de elétrons. A partir dos dados obtidos após tratamento térmico e irradiação, conclui-se que $\mathrm{Mn}^{2+}$ é o principal centro cromóforo da cor rosa, favorecido por um certo grau de desordem presente na estrutura dessa espécie. A irradiação intensifica a cor rosa pálida, enquanto o aquecimento a $450^{\circ} \mathrm{C}$ descolore o rosa.

\section{INTRODUÇÃO}

As turmalinas são conhecidas como gemas que apresentam grande variedade de cores e composições químicas, principalmente quando se trata daquelas provenientes de pegmatitos graníticos. Em diferentes zonas dos pegmatitos, encontram-se cristais com uma gama de tonalidades que vão do negro até o incolor. Investigações químicas sobre causas de cor nesses minerais, feitas isoladamente, não permitem correlacionar a cor com as estruturas microscópicas de defeitos pontuais, os quais podem estruturar um centro de cor. O conhecimento sobre centros cromóforos criados por irradiação ou impurezas de metais de transição, no grupo da turmalina, é escasso e explicado somente a partir de medidas ópticas interpretadas por intermédio da teoria do campo cristalino. Investigações mais profundas sobre defeitos pontuais por técnicas de ressonância magnética são pouco conhecidas. Lima et al (2003) confirmam, por meio de espectrometria fotoacústica, que elementos em quantidades traços podem ser responsáveis por uma variedade de cores e centros de cores em turmalinas. Tem-se conhecimento que centros de cor do tipo O- (Bershov et al. 1969) e OAl-O (Krambrock et al. 2002) foram estabelecidos em turmalinas rosas e amarelas, respectivamente. Além disso, alguns trabalhos sugerem que em turmalinas ricas em manganês (8-9\% $\mathrm{MnO})$ que apresentam cores variando do rosa ao marrom amarelado, seus matizes são devidos a diferentes intensidades de radiação natural de $\mathrm{Mn}^{2+}$ inicial para $\mathrm{Mn}^{3+}$ (Ertl et al. 2003). Entretanto, turmalinas rosas que apresentam quantidades menores de manganês ainda há controvérsias quanto da atribuições das bandas de absorção óptica e centros paramagnéticos induzidos ou não por irradiação. 
Por esses motivos, o principal objetivo do presente estudo é correlacionar as bandas de absorção óptica com as informações estruturais dos centros cromóforos, principalmente simetria, intensidade do campo cristalino, identidade química dos ligantes e a presença de fenômenos de transferência de carga. Para correlacionar a estrutura microscópica de um defeito com bandas de absorção, foram utilizadas espectroscopia óptica (UVvisível), ressonância paramagnética eletrônica (EPR) e detecção óptica da ressonância magnética (ODMR ou ODEPR) por meio do dicroísmo circular magnético de absorção (MCDA). A ressonância paramagnética eletrônica fornece melhor entendimento dos defeitos estruturais, ao passo que ressonância magnética óptica pode dar correlação direta entre o espectro óptico (cor) e o modelo microscópico dos centros de cor. Além disso, foram relaizadas microanálises por meio de microssonda eletrônica.

Foram estudadas turmalinas provenientes de pegmatitos do Campo Pegmatítico de Coronel MurtaVirgem da Lapa-Rubelita, parte integrante do Distrito Pegmatítico de Araçuaí, situado na porção nordeste do Estado de Minas Gerais.

\section{MÉTODOS UTILIZADOS}

As amostras foram selecionadas com base na homogeneidade macroscópica da cor. Foram confeccionadas lâminas polidas para análise de microssonda eletrônica e lâminas bipolidas $(5 \times 3 \times 2 \mathrm{~mm})$, perpendiculares e paralelas ao eixo $\mathbf{c}_{0}$, utilizadas nas demais técnicas espectroscópicas. Os espectros foram obtidos inicialmente com amostras naturais e posteriormente foram analisados os efeitos da irradiação.

Análises pontuais nos cristais foram realizadas em microssonda eletrônica, no laboratório do Instituto de Geociências da Universidade de Brasília utilizando-se equipamento CAMEBAX SX-50 (1990) com 4 espectrômetros WDS e 1 EDS. As condições de operação para todos os elementos foram de $15 \mathrm{KV}$ e 20 nA. Foram utilizados os padrões: jadeíta $(\mathrm{Na})$, ortoclásio (K), hortolita (Si, Fe, Mg, Mn), fluorita (F), TiO 2 (Ti), anortita $(\mathrm{Ca}, \mathrm{Al}), \operatorname{cromita}(\mathrm{Cr}), \mathrm{NiO}(\mathrm{Ni}), \mathrm{ZnO}(\mathrm{Zn}), \mathrm{V}_{2} \mathrm{O}_{3}$ (V) e $\mathrm{Cu}_{2} \mathrm{O}(\mathrm{Cu})$.

Os dados químicos para cada cristal correspondem a 20 microanálises medidas ao longo do cristal. As turmalinas são expressas pela fórmula geral $\mathrm{XY}_{3} \mathrm{Z}_{6}\left(\mathrm{BO}_{3}\right)_{3}\left(\mathrm{~T}_{6} \mathrm{O}_{18}\right) \mathrm{V}_{3} \mathrm{~W}$ (Hawthorne \& Henry 1999), onde $\mathrm{X}=\mathrm{Na}^{+}, \mathrm{Ca}^{2+}, \mathrm{K}^{+}, \mathrm{Mg}^{2+}$ ou ainda pode manter-se vazio; $\mathrm{Y}=\mathrm{Fe}^{2+}, \mathrm{Mg}^{2+}, \mathrm{Al}^{3+}, \mathrm{Li}^{+}, \mathrm{Fe}^{3+}, \mathrm{Mn}^{2+}, \mathrm{V}^{3+}, \mathrm{Cr}^{3+}$, $\mathrm{Ti}^{4+} ; \mathrm{Z}=\mathrm{Al}^{3+}, \mathrm{Fe}^{3+}, \mathrm{Mg}^{2+}, \mathrm{Cr}^{3+} \mathrm{eV}^{3+} ; \mathrm{T}=\mathrm{Si} 4+, \mathrm{Al} 3+; \mathrm{V}^{\mathrm{o}}$ $\mathrm{OH}, \mathrm{O}^{2} \mathrm{e} \mathrm{W}^{\mathrm{o}} \mathrm{OH}, \mathrm{F}^{-}, \mathrm{O}^{2-}$. As fórmulas estruturais foram calculadas com base em 24,5 ânions, assumindo

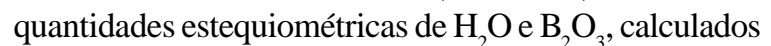
a partir de $\mathrm{OH}^{-}=(4-\mathrm{F})=4$ átomos por fórmula unitária (apfu) e $\mathrm{B}^{3+}=3 \mathrm{apfu}$, respectivamente. A quantidade de $\mathrm{Li}^{+}$atribuída ao sítio cristalográfico $\mathrm{Y}$ foi feita como sendo igual ao somatório ideal do sítio Y (=3) menos a quantidade dos outros cátions que ocupam o mesmo sítio, ou seja Li = 3-Y (Burns et al. 1994). O Mn foi considerado como $\mathrm{Mn}^{2+}$ a partir dos resultados de EPR. As atribuições dos sítios foram feitas, assumindo que: 1) a deficiência de $\mathrm{Si}$ no sítio $\mathrm{T}$ é compensada por $\mathrm{Al}^{[4]}$; 2) o Z é ocupado por $\mathrm{Al}^{[6]}$; 3) os cátions restantes, com exceção de $\mathrm{Na}$, Ca e K no sítio X, ocupam o sítio Y.

As análises por espectroscopia de Ressonância Paramagnética Eletrônica (EPR) convencional foram desenvolvida no Laboratório de EPR do Departamento de Física, Instituto de Ciências Exatas da Universidade Federal de Minas Gerais e no Departamento de Física da Universidade de Paderborn, Alemanha, utilizando espectrômetros com cavidade ressonante cilíndrica $\left(\mathrm{TE}_{011}\right)$, operando com frequiências de microondas de 9 a $11 \mathrm{GHz}, 500 \mathrm{~mW}$ Klystron (VARIAN) e campo magnético (B) 0-0.8T. Para aumentar o sinal foi aplicada a modulação de campo de $100 \mathrm{kHz}$ e detecção lock-in (EG\&G Princeton). Foram medidos espectros com B paralelo e perpendicular ao eixo $\mathbf{c}_{\mathbf{0}}$, em temperatura ambiente, e utilizado criosystem de fluxo de He para temperaturas entre 4 e $300 \mathrm{~K}$.

A detecção óptica da ressonância magnética foi realizada no Laboratório do Departamento de Física da Universidade de Paderborn, Alemanha, utilizando espectrômetros com cavidade ressonante cilíndrica $\left(\mathrm{TE}_{011}\right)$, operando com freqüências de microondas de 24,02 GHz. Foram medidos espectros com campo magnético (B) paralelo e perpendicular ao eixo $\mathbf{c}_{0}$, utilizando criosystem de fluxo de He para temperaturas entre 1.5 e $300 \mathrm{~K}$. Os ensaios de dicroísmo circular magnético de absorção óptica foram realizado no Laboratório do Departamento de Física da Universidade de Paderborn, Alemanha, no qual o espectro de excitação da ODEPR foi medido modulando-se a amplitude da microonda.

As medidas de absorção óptica foram realizadas em um espectrômetro modelo Hitachi U-3501 à temperatura ambiente, com um dispositivo especial para medida de absorção em sólidos, em rotina no laboratório do CDTN/ CNEN, Belo Horizonte. Para as medidas, as amostras previamente preparadas foram parcialmente cobertas com fita opaca criando fendas de aproximadamente $1 \mathrm{~mm}$ para a passagem do feixe de luz. Colocou-se um polarizador entre a amostra e a fonte luminosa, de forma que a absorção medida está relacionada com a orientação cristalográfica da amostra. Estas medidas permitiram uma caracterização precisa da cor da amostra assim como mostrou quais comprimentos de onda estão sendo absorvidos na faixa visível do espectro eletromagnético.

Os espectros Raman polarizados em monocristais foram obtidos no Laboratório de Micro-Raman do Departamento de Física da Universidade Federal de Minas Gerais em um espectrômetro DILOR X-Y. As linhas 488 e/ou $514 \mathrm{~nm}$ de um laser de Ar foram usadas na excitação. Os espectros a temperatura ambiente foram obtidos em geometria de backscattering. O raio laser foi focalizado em um diâmetro de $2 \mathrm{~mm}$ com objetiva Zeiss de 80X. A maioria dos espectros foi medida com potência de $10,6 \times 25 \mathrm{~mW}$. Os espectros experimentais foram ajustados com linhas Gaussianas. Os espectros 
foram ajustados com linhas de igual largura a meiaaltura. Foi utilizado o fator $r^{2}(\sim 0,99)$ para testar o modelo ajustado.

Os experimentos de irradiação foram feitos na Companhia Brasileira de Esterilização (CBE) em São Paulo, onde as amostras foram irradiadas em célula de raios gama com fonte de cobalto-60. Foram utilizados doses de $5 \mathrm{MGy}$ de raios gamas equivalente a 250 horas de exposição (20 KgGy/h) em temperatura ambiente. As amostras, após a irradiação, não ficaram radioativas.

\section{CARACTERIZAÇÃOQUÍMICA EESTRUTURAL}

Os cristais apresentam cores naturais pálidas a intensas e, na maioria das vezes, possuem qualidade gemológica. Diversas tonalidades esbranquiçadas foram amostradas e consideradas padrões, assim como diversos tons de rosa, do mais pálido até o mais intenso.
A Tabela 1 apresenta a composição química dada pela média das análises em cada cristal, bem como os valores referentes à fórmula estrutural. Os elementos $\mathrm{Si}, \mathrm{Al}, \mathrm{Fe}$, $\mathrm{Mn}, \mathrm{Mg}, \mathrm{Zn}, \mathrm{Ti}, \mathrm{Li}^{*}$, Na, Ca, K, OH e F foram considerados maiores e subordinados, os demais são elementos traço (*estequiométrico).

As amostras estudadas correspondem a membros finais que apresentam pouca variação em relação à fórmula ideal da molécula da elbaíta, com exceção dos teores de manganês (Tab. 1), cuja caracterização cristaloquímica é um dos objetivos. O conteúdo desse elemento está diretamente relacionado com a intensidade da cor rosa; quanto mais escuro o matiz, maior é o teor do manganês. O ferro está presente em conteúdos-traço, dificultando sua caracterização. Os teores de $\mathrm{SiO}_{2}$ e $\mathrm{Al}_{2} \mathrm{O}_{3}$ estão coerentes com a composição química ideal desses minerais, assim como de álcalis e cálcio. A avaliação dos dados químicos é concordante com as

Tabela 1 - Média das análises químicas das turmalinas estudadas e suas fórmulas estruturais.

\begin{tabular}{|c|c|c|c|c|c|}
\hline & \multirow{2}{*}{\begin{tabular}{|c|} 
Rosa escuro \\
MC
\end{tabular}} & \multicolumn{2}{|c|}{ Rosa pálido } & \multirow{2}{*}{$\begin{array}{l}\text { Rosa } \\
\text { MR1 } \\
\end{array}$} & \multirow{2}{*}{$\begin{array}{c}\text { Branca } \\
\text { U1 }\end{array}$} \\
\hline & & MR3 & MR2 & & \\
\hline$\overline{\mathrm{SiO}_{2}}$ & 37,93 & $\begin{array}{l}38,02 \\
\end{array}$ & $\begin{array}{l}38,86 \\
\end{array}$ & $\begin{array}{l}39,04 \\
\end{array}$ & $\begin{array}{l}38,35 \\
\end{array}$ \\
\hline $\mathrm{TiO}_{2}$ & 0 & 0,01 & 0,01 & 0 & 0,01 \\
\hline $\mathrm{Al}_{2} \mathrm{O}_{3}$ & 43,98 & 44,16 & 42,89 & 41,97 & 44,42 \\
\hline $\mathrm{FeO}$ & 0,01 & 0,02 & 0,04 & 0,15 & 0,01 \\
\hline $\mathrm{Fe}_{2} \mathrm{O}_{3}$ & 0 & 0 & 0 & 0 & 0 \\
\hline $\mathrm{MgO}$ & 0 & 0 & 0 & 0 & 0 \\
\hline MnO & 0,52 & 0,25 & 0,17 & 0,12 & 0,02 \\
\hline $\mathrm{ZnO}$ & 0,02 & 0,02 & 0,01 & 0,04 & 0,07 \\
\hline $\mathrm{CaO}$ & 0,23 & 0,41 & 0,29 & 0,4 & 0,1 \\
\hline $\mathrm{Na}_{2} \mathrm{O}$ & 1,89 & 1,63 & 1,67 & 2,55 & 2,2 \\
\hline $\mathrm{K}_{2} \mathrm{O}$ & 0,01 & 0,01 & 0,01 & 0,01 & 0,01 \\
\hline $\mathrm{Li}_{2} \mathrm{O}$ & 1,18 & 1,26 & 1,62 & 1,91 & 1,21 \\
\hline $\mathrm{H}_{2} \mathrm{O}^{+}$ & 3,79 & 3,56 & 3,54 & 3,49 & 3,65 \\
\hline $\mathbf{F}$ & 0,01 & 0,53 & 0,57 & 0,7 & 0,38 \\
\hline $\mathrm{O}=\mathrm{F}$ & 0 & 0,22 & 0,24 & 0,29 & 0,16 \\
\hline TOTAL & 89,57 & 89,67 & 89,46 & 90,09 & 90,58 \\
\hline $\mathrm{Si}^{4+}$ & 5,99 & 5,98 & 6,1 & 5,94 & 5,99 \\
\hline $\mathrm{Al}^{3+}$ & 0,02 & 0,02 & 0 & 0,08 & 0,01 \\
\hline$T S i$ & 6,01 & 6 & 6,1 & 6,02 & 6 \\
\hline $\mathrm{Al}^{3+}$ & 6 & 6 & 6 & 6 & 6 \\
\hline$T Z$ & 6 & 6 & 6 & 6 & 6 \\
\hline $\mathrm{Fe}^{+2}$ & 0 & 0 & 0,01 & 0,78 & 0 \\
\hline $\mathrm{Fe}^{3+}$ & 0 & 0 & 0 & 0 & 0 \\
\hline $\mathrm{Mg}^{2+}$ & 0 & 0 & 0 & 0,05 & 0 \\
\hline $\mathrm{Mn}^{2+}$ & 0,07 & 0,03 & 0,02 & 0,13 & 0 \\
\hline $\mathrm{Ti}^{4+}$ & 0 & 0 & 0 & 0,01 & 0 \\
\hline $\mathrm{Zn}^{2+}$ & 0 & 0 & 0 & 0,03 & 0,01 \\
\hline $\mathrm{Li}^{+}$ & 0,75 & 0,8 & 1,03 & 0,75 & 0,76 \\
\hline $\mathrm{Al}^{3+}$ & 2,17 & 2,16 & 1,94 & 1,21 & 2,22 \\
\hline$T Y$ & 3 & 3 & 3 & 3 & 3 \\
\hline $\mathrm{Ca}^{2+}$ & 0,04 & 0,07 & 0,05 & 0,05 & 0,02 \\
\hline $\mathrm{Na}^{+}$ & 0,58 & 0,5 & 0,51 & 0,8 & 0,67 \\
\hline $\mathrm{K}^{+}$ & 0 & 0 & 0 & 0,01 & 0 \\
\hline$T X$ & 0,62 & 0,57 & 0,56 & 0,86 & 0,68 \\
\hline $\mathrm{OH}^{-}$ & 4 & 3,73 & 3,72 & 3,79 & 3,81 \\
\hline$F^{-}$ & 0 & 0,27 & 0,28 & 0,21 & 0,19 \\
\hline $\mathrm{TOH}$ & 4 & 4 & 4 & 4 & 4 \\
\hline
\end{tabular}


dimensões da cela unitária $\left(\mathbf{a}_{\mathbf{0}}=15,768 \AA \mathbf{c}=7,077 \AA\right)$, segundo os quais todas as turmalinas analisadas são moléculas muito próximas ao membro-final elbaíta.

Para a caracterização do manganês, optou-se também pela utilização da espectroscopia de ressonância paramagnética eletrônica (EPR), técnica que permite detectar mudanças nos níveis eletrônicos dos defeitos paramagnéticos na estrutura cristalina, correlacionados aos centros de cor, sejam produzidos por irradiação ou por íons cromóforos em concentrações tão pequenas como da ordem de ppm. Existem diversas discussões sobre espectros EPR de íons $\mathrm{Mn}^{2+}$ ( spin nuclear $\mathrm{I}=5 / 2$ ) em minerais observados a temperatura ambiente (De Biasi \& Fernandes 1984; McGavin et al. 1982; Shaffer et al. 1976; Holuj \& Manoogian 1968). Espera-se observar, no mínimo, 6 linhas $(I=5 / 2)$ de igual intensidade devido aos componentes da estrutura hiperfina (HFS), divididas em cinco pacotes em função dos componentes das estruturas finas ( $5 \times 6=30$ linhas) para orientações arbitrárias. Distorções locais do sítio do $\mathrm{Mn}^{2+}$ podem explicar variações nos componentes HFS, assim como desvios da simetria (espectros anisotrópicos). Além disso, a largura das linhas pode aumentar consideravelmente com o aumento da concentração. Transições proibidas fracas (DI $= \pm 1$ ) ocorrem entre os picos mais intensos e podem ter suas intensidades relativas também alteradas pelo efeito da distorção (Calas 1988).

$\mathrm{O}$ espectro EPR convencional registrado em

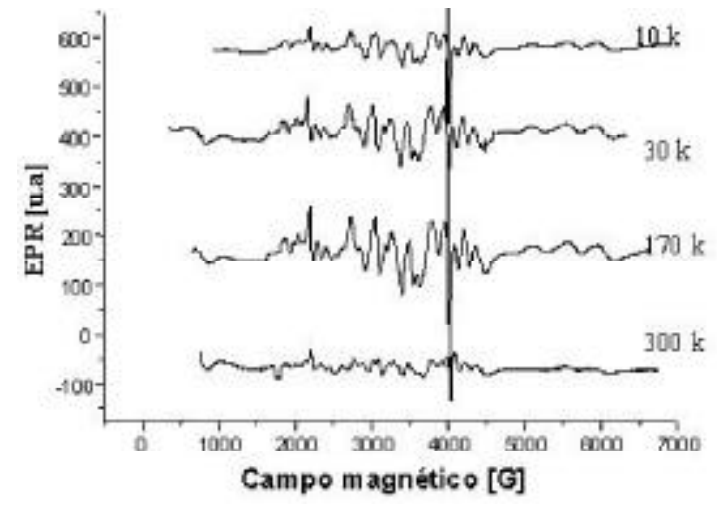

Figura 1-EPR da amostra MR3, antes da irradiação, em diferentes temperaturas e campo magnético externo $\mathrm{B} / / \mathrm{c}$.

diferentes temperaturas de uma das elbaítas, antes da irradiação, é ilustrado na figura 1. Nesse espectro, as seis linhas originadas a partir do spin nuclear do $\mathrm{Mn}^{2+}$ em sítio octaédrico foram diagnosticadas, e pertencem à transição de Zeeman $-5 / 2 \rightarrow+5 / 2$, $-3 / 2 \rightarrow+3 / 2$ e $-1 / 2 \rightarrow+1 / 2$, respectivamente. Linhas intermediárias podem ser decorrentes de transições proibidas fracas, e são melhores resolvidas a baixas temperaturas. Além disso, o aspecto geral do espectro mostra claramente que o $\mathrm{Mn}^{2+}$ está localizado em um sítio octaédrico distorcido devido ao alargamento das linhas e é o único centro paramagnético da elbaíta rosa natural.
Após a irradiação, a amostra MR3, originalmente rosa pálida, adquiriu tonalidade rosa mais escuro. Nos espectros EPR, além do $\mathrm{Mn}^{2+}$ foram identificados mais três centros paramagnéticos, $\mathrm{Fe}^{3+}, \mathrm{H}^{\mathrm{o}}$ e um centro preliminarmente sem diagnóstico denominado de centro A (Fig. 2). A identificação do $\mathrm{Fe}^{3+} \mathrm{e}$ do átomo de hidrogênio $\left(\mathrm{H}^{\circ}\right)$ é direta em relação a forte interação

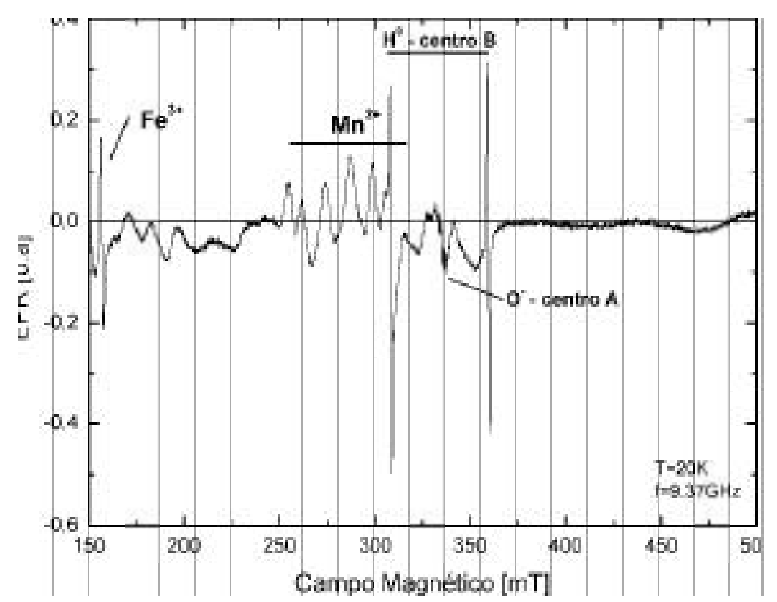

Figura 2 - Espectro EPR da amostra MR3 após girradiação a $20 \mathrm{~K}$ e freqüências de 9,37 GHz.

hiperfina isotrópica. Por outro lado, o centro A parece estar relacionado com um centro do tipo $\mathrm{O}^{-}$, apesar de não ter sido observado interação hiperfina com $17^{\circ}$ (spin nuclear I=5/2 e abundância natural de $0,037 \%$ ).

$\mathrm{O} \mathrm{Fe}{ }^{3+}$ identificado apenas após a irradiação provavelmente atua como um compensador de carga e/ ou estabilizador do centro de cor, ou ainda como um causador de tonalidade adicional. Entretanto, essas hipóteses são mais bem elaboradas por intermédio da caracterização de bandas de absorção no MCDA total (dicroísmo circular magnético de absorção) e do espectro de excitação do ODEPR (detecção óptica do EPR) dessas elbaítas, conforme particularmente registrado na amostra MR3, antes da irradiação.

Centros similares ao centro $\mathbf{A}$ foram observados em elbaítas rosas e incolores por Bershov et al. (1969) e Krambrock et al. (2002), respectivamente. Os primeiros concluiram, através do EPR, absorção óptica e luminescência, que a elbaíta de cor rosa com banda de absorção centrada a $515 \mathrm{~nm}$ é devido a interação do Ocom dois núcleos de $\mathrm{Al}$ equivalentes. Os segundos concluiram que a cor amarela da elbaíta é devida a centro buraco (hole-center) do tipo $\mathrm{Al}-\mathrm{O}^{-}-\mathrm{Al}$, induzido por irradiação dentro de campos cristalinos intermediários a fortes. De fato, os centros de aprisionamento de $\mathrm{O}^{-}$ têm sido extensivamente investigados com técnicas de ressonância magnética em a- $\mathrm{SiO}_{2}$ ricos em $\mathrm{Al}$ irradiados com raios gama, formando um par de $\mathrm{Al}^{-} \mathrm{O}^{-}$(Pinheiro et al. 1999). A hipótese dos primeiros autores está descartada e sua conclusão é inconsistent, uma vez que pode existir uma superposição de no mínimo duas bandas de absorção relacionada com defeitos, uma amarela (Krambrock et al. 2002) e outra rosa (Mn2+, neste trabalho). 


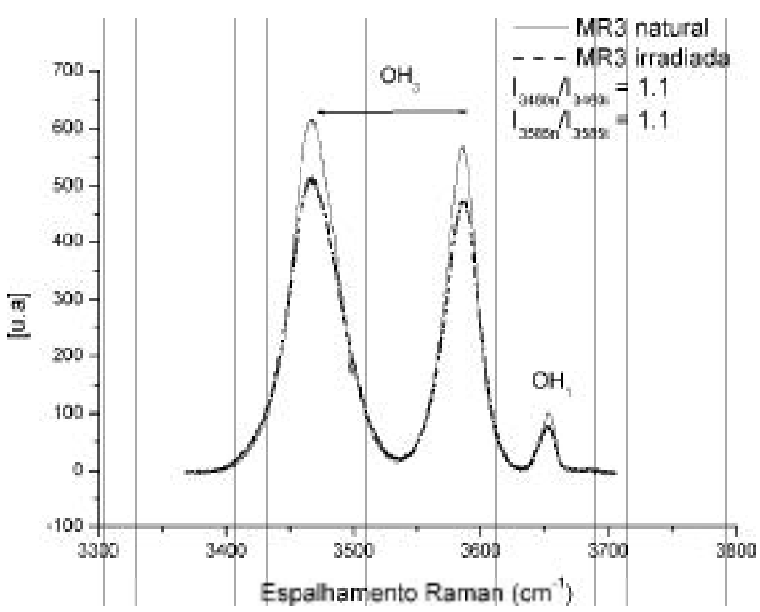

Figura 3 - Espectros Raman da amostra MR3 antes e após irradiação. As intensidades relativas das bandas atribuídas ao estiramento do grupo $\mathrm{OH}_{3}$ decrescem após a irradiação na razão $\sim 1$.

$$
I_{3460}=\text { intensidade relativa. }
$$

Baseado nos argumentos acima, propõe-se que o centro A é um centro Al-O- ${ }^{-}$-Al. Neste sentido, levando em consideração os espectros Raman (Fig. 3) das elbaítas rosas, antes e após irradiação, observa-se que as intensidades relativas das bandas atribuídas ao estiramento do grupo $\mathrm{OH}_{3}$ (Castañeda et al. 2000) decrescem após a irradiação na razão próxima a 1 . Deve-se destacar que os grupos $\mathrm{OH}$ na estrutura das
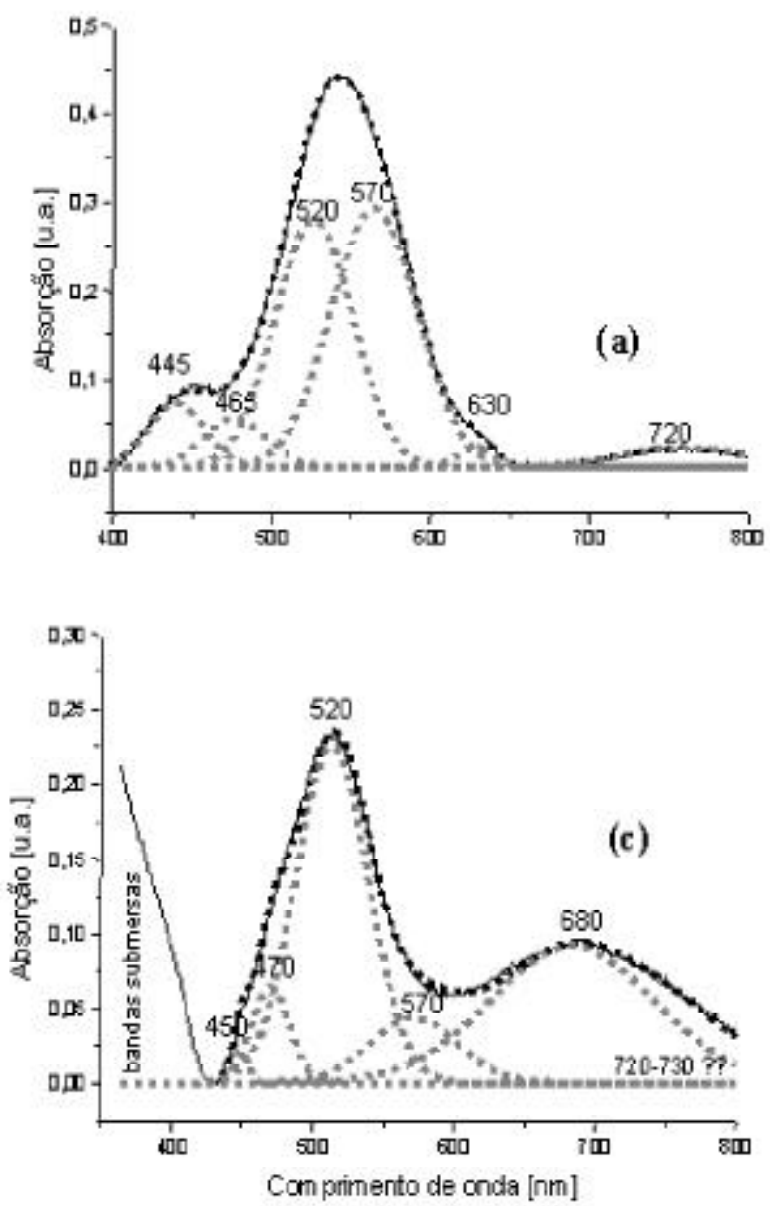

turmalinas estão rodeados pelos sítios $\mathrm{Y}$ e Z, sendo afetados diretamente pelo ambiente local. Além disso, espectros Raman de uma amostra rosa escuro (MC), que após irradiação não apresentou intensificação da cor nem registro do centro Ho, também não apresentam diferença nas razões das bandas de estiramento do grupo $\mathrm{OH}_{3}$.

Considerando os dois $\mathrm{Al}$ vizinhos pela coordenação do ambiente local das bandas de estiramento do grupo $\mathrm{OH}_{3}$, pode-se propor um provável processo de criação do centro O-, em que ele é formado por irradiação gama no sítio $\mathrm{OH}_{3}$, que apresenta dois $\mathrm{Al}$ como vizinhos próximos de acordo com o esquema: $\mathrm{Al}-\mathrm{OH}^{-}-\mathrm{Al}+\mathrm{g} \rightarrow$ $\mathrm{Al}-\mathrm{O}^{-}-\mathrm{Al}$ (hole trap) $+\mathrm{H}^{\mathrm{o}}$ (electron trap) .

\section{CARACTERIZAÇÃOÓPTICA}

Os espectros de absorção óptica foram obtidos nas amostras com a luz propagando-se paralela aos eixos $\mathbf{c}_{\mathbf{0}}$ e $\mathbf{a}_{\mathbf{0}}(\mathrm{E} \| \mathrm{c}$ e E1c). Para melhor exatidão do comprimento de onda e da quantidade de bandas, uma vez que podem ocorrer superposições de bandas, foram feitos ajustes utilizando modelo com linhas gaussianas e sem restrição na maioria dos espectros. Nas turmalinas rosas estão presentes quatro bandas dominantes centradas a 450 , 470,520 e $570 \mathrm{~nm}$ e uma banda larga, porém duvidosa, em regiões acima de $720 \mathrm{~nm}$. Naquelas de matizes mais
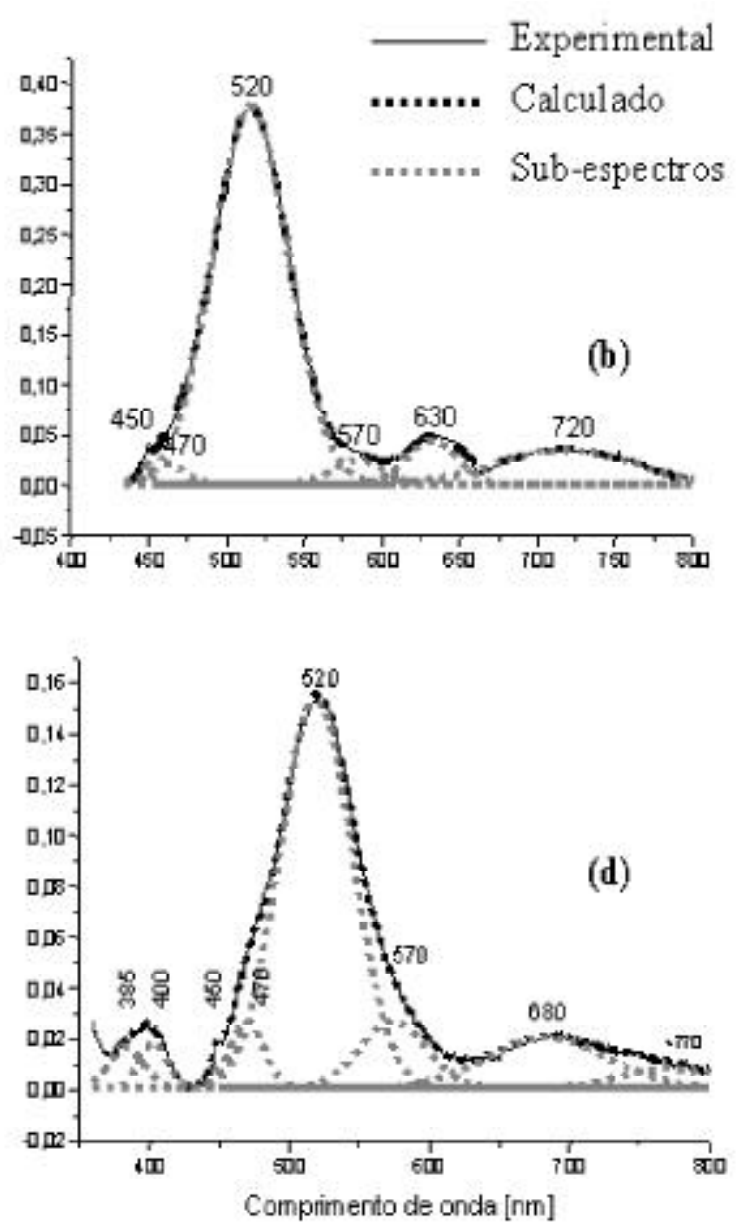

Figura 4 - Espectro de absorção óptica das elbaítas rosas. $M C=$ rosa intenso (a), MR2 = rosa pálido amarelada (b), MR3 = rosa pálido $E^{\wedge} c(c)$ e MR3 E\|c (d). 
amarelados (MR2) observa-se uma banda proeminente centrada a $630 \mathrm{~nm}$, ao passo que nas rosas pálidas (MR3) tem-se a presença da banda centrada a $680 \mathrm{~nm}$ e mais duas subordinadas a polarização à $385 \mathrm{e} \sim 400 \mathrm{~nm}$. A figura 4 apresenta os espectros de absorção óptica das amostras MC, MR2 e MR3.

A figura 5 apresenta os espectros de absorção óptica da amostra MR3 (rosa pálida natural) após a irradiação, ao aquecimento à $450^{\circ} \mathrm{C}$ e ao aquecimento + irradiação. Após irradiação, a qual produz matizes mais escuros do rosa, ocorre a produção da banda centrada a $630 \mathrm{~nm}$ e incremento das bandas centradas a $520 \mathrm{~nm}$. Por outro lado, após aquecimento a $450^{\circ} \mathrm{C}$, em que a amostra apresenta-se próxima a incolor, a maioria das bandas quase desaparecem concomitante ao desaparecimento

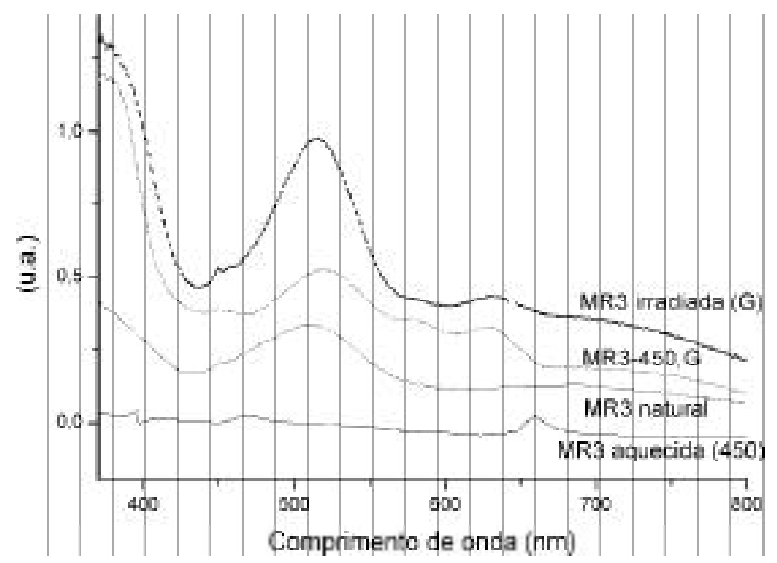

Figura 5 - Espectro de absorção óptica da amostra MR3 antes e após tratamentos por irradiação e térmico.

da cor rosa. Quando a amostra é submetida ao aquecimento anterior à irradiação, a cor produzida é muito semelhante àquela das amostras naturais. As bandas centradas a $520 \mathrm{~nm}$ apresentam-se levemente afetadas e concomitante ao aparecimento da banda centrada a $630 \mathrm{~nm}$.

Melhor definição dos espectros ópticos foram observados no dicroísmo circular magnético de absorção total (MCDA) da amostra MR3 (Fig. 6), a qual apresenta três bandas ópticas largas centradas em 680,

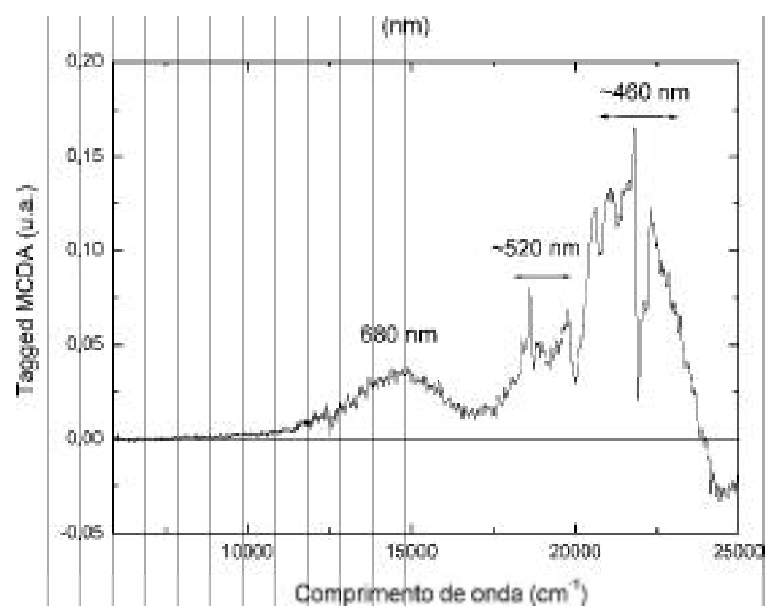

Figura 6 - Dicroísmo circular magnético de absorção (MCDA) da amostra rosa MR3. $\sim 520$ e $460 \mathrm{~nm}$. As duas últimas bandas apresentam linhas adicionais, intermediárias, finas e juntas, típicas de transições $d$ - $d$ (Burns 1993). O primeiro pacote apresenta bandas intermediárias no intervalo entre 540 e $500 \mathrm{~nm}$, e o segundo, entre 480 e $440 \mathrm{~nm}$. A banda centrada a $680 \mathrm{~nm}$, em energia maior, apresenta-se larga e de baixa intensidade em relação aos outros dois conjuntos.

A Figura 7 apresenta o espectro de excitação do ODEPR, medido na banda de absorção do MCDA total a 1,5 K. Dois pacotes de linhas de EPR na forma de absorção são observados e comparáveis ao espectro de EPR convencional, o qual está relacionado ao centro paramagnético do $\mathrm{Mn}^{2+}$. Um deles apresenta tensor $\mathrm{g} 4$, enquanto o outro, $g \sim 2$ (tensor $g$ descreve a interação magnética entre os momentos de dipolo do spin e orbital e reflete a simetria do defeito e de sua vizinhaça). Esse último apresenta intensidade menor que o primeiro. Brodbeck \& Buckrey (1981) e Kliava (1986) descrevem

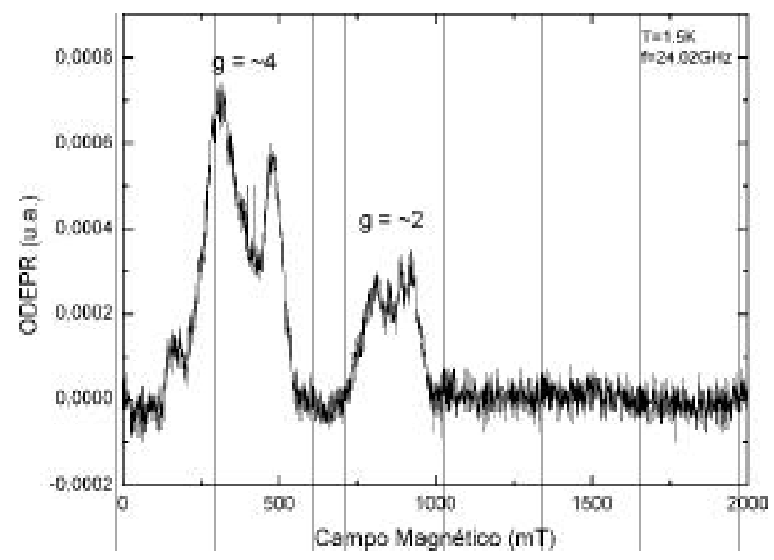

Figura 7 - Espectro de ODEPR da amostra MR3, antes da irradiação, em temperatura de $1,5 \mathrm{Ke}$ freqüência de 24,02 GHz, identificando o centro paramagnético do $\mathrm{Mn}^{2+}$.

modelos para distinguir a coordenação dos íons $\mathrm{Mn}^{2+} \mathrm{e}$ $\mathrm{Fe}^{3+}$ em quartzo, por intermédio do padrão da distribuição bidimensional dos parâmetros D e E descritos nos espectros de EPR. A relação de tal distribuição distingue dois sítios não equivalentes para ambos metais, um relativo a $g=4,3$ e outro a $g=2$. Alto grau de desordem (short -range) também é caracterizado para o sítio com $g=4,3$.

Considerando a hipótese descrita, pode-se supor que o $\mathrm{Mn}^{2+}$, presente na amostra rosa, está localizado em dois sítios octaédricos Y distintos, magneticamente não equivalentes, designados, arbitrariamente Y1 e Y2. Além disso, os espectros foram obtidos em $\mathrm{B} \| \mathbf{c}$, por isso era de se esperar que o somatório de três sítios $\mathrm{Y}$ equivalentes registrasse apenas um único sinal. A presença de dois sinais, pode, de fato, diagnosticar a presença de dois sítios Y magneticamente não equivalentes do manganês. Deve-se destacar, também, a hipótese de que o pacote das linhas em $\mathrm{g}=4$ pode originar-se por transições proibidas com Dms $= \pm 2$, 
apresentando-se fortes, devido à temperatura de 1,5K. O dicroísmo circular magnético de absorção do espectro de excitação ODEPR é idêntico ao MCDA total. Como o único centro paramagnético registrado no ODEPR é do $\mathrm{Mn}^{2+}$, conclui-se que esse é o principal e único centro de cor nas elbaítas rosas. O ferro registrado nas microanálises não apresenta banda de absorção no MCDA e, portanto, não contribui para a cor. Todas as bandas observadas na faixa do visível no espectro de absorção óptica (vide fig. 2) são similares às apresentadas no MCDA, o que pode, então, ser atribuídas às interações do manganês. Além disso, bandas $d$ - $d$ adicionais em baixa e alta energia podem estar superpostas ou submersas no sistema de bandas do NIR e na margem do UV, respectivamente, como visto nos espectros ajustados.

É de se esperar que a presença de $\mathrm{Mn}^{2+}$ em sítios $\mathrm{Y}$ magneticamente não equivalentes cause um certo grau de desordem na estrutura da elbaíta, uma vez que um alto grau de ordenamento dos cátions implica em baixo número de sítios Y não equivalentes (Oliveira et al. 2002). Partindo dessa premissa, foi avaliado o ambiente local do sítio Y por intermédio da espectroscopia Raman.

O comportamento espectral da região do estiramento O-H é discutido em termos de ambiente local e grau de cristalinidade, com o objetivo de elucidar os fenômenos de ordem e desordem entre as amostras. O estiramento do grupo $\mathrm{OH}$ é muito sensível à ocupação do sítio em relação aos íons que estão nos arredores e pode, portanto, mostrar relações entre os modos ativos e os fenômenos de ordem-desordem. As bandas relativas ao estiramento $\mathrm{O}-\mathrm{H}$ são normalmente observadas na região entre 3.800 a $3.000 \mathrm{~cm}^{-1}$ e são ativas no Raman. $\mathrm{O}$ íon $\mathrm{OH}^{-}$é linear, assimétrico e pertence ao grupo pontual $\mathrm{C}_{V}$, o qual tem uma vibração fundamental, $\Gamma=\mathbf{A}_{1}$, que deriva do estiramento da ligação $\mathrm{O}-\mathrm{H}$, cuja notação usual é $v_{O}-{ }_{H}$. Os números de onda vibracionais dessa banda são fortemente relacionados à natureza das ligações químicas e as estruturas geométricas envolvidas. Por isso, duas espécies minerais nunca terão o mesmo espectro.

$\mathrm{Na}$ estrutura da turmalina, os grupos $\mathrm{OH}^{-}$podem ocupar duas diferentes posições. A primeira posição, denominada $\mathrm{OH}_{1}$, localiza-se no centro do anel hexagonal e ocupa um sítio de simetria $\mathrm{C}_{3 \mathrm{~V}}$, e tem seu oxigênio coordenado a três cátions do sítio Y. O segundo $\mathrm{OH}^{-}$, é denominado de $\mathrm{OH}_{3}$, localiza-se na borda do anel, ocupa o sítio de simetria $\mathrm{C}_{\mathrm{S}}$ e tem seu oxigênio coordenado a um cátion do sítio $\mathrm{Y}$ e a dois cátions do $\mathrm{Z}$.

Castañeda et al. (2000) estudando a série elbaítaschorlita no espectro no infravermelho e por meio da análise de grupo de fator, definiram três bandas do estiramento $\mathrm{OH}$, uma relacionada ao $\mathrm{OH}_{1}$ e duas ao $\mathrm{OH}_{3}$. Esses autores observaram dois comportamentos espectrais para as turmalinas dessa série: no primeiro, o número de bandas está de acordo com aquelas previstas pela análise do grupo de fator, mas ocorre desvio dos números de onda das bandas e seu alargamento, devido à substituição progressiva de elementos com maior eletronegatividade $\left(\mathrm{Fe}^{2+}\right)$ pelos de menor $\left(\mathrm{Li}^{+}\right)$, ao longo da série; e no segundo, há uma relação entre o desdobramento das bandas, particularmente do estiramento do grupo $\mathrm{OH}_{1}$ de membros intermediários. Oliveira et al. (2002), reinterpretaram esses dados e estenderam o estudo a outras espécies do grupo da turmalina. Por intermédio da integração de dados de MS, os autores caracterizaram o grau de ordemdesordem através da forma e do número de bandas do estiramento $\mathrm{OH}$.

Estudos do espectro Raman em turmalinas são mais escassos e apresentam apenas os modos de vibração da rede, em números de onda baixos (Huang 1999; Gasharova et al. 1997). Esses trabalhos apenas classificaram o tipo de molécula ou a série a que as turmalinas pertencem, por meio da integração dos dados químicos com o espectro Raman.

A figura 8 apresenta o espectro Raman ajustado da elbaíta rosa MR3, idêntico aos das demais amostras rosa deste trabalho. Todos os espectros foram ajustados com linhas gaussianas e apresentam fator $\mathbf{r}^{2}$ próximo a

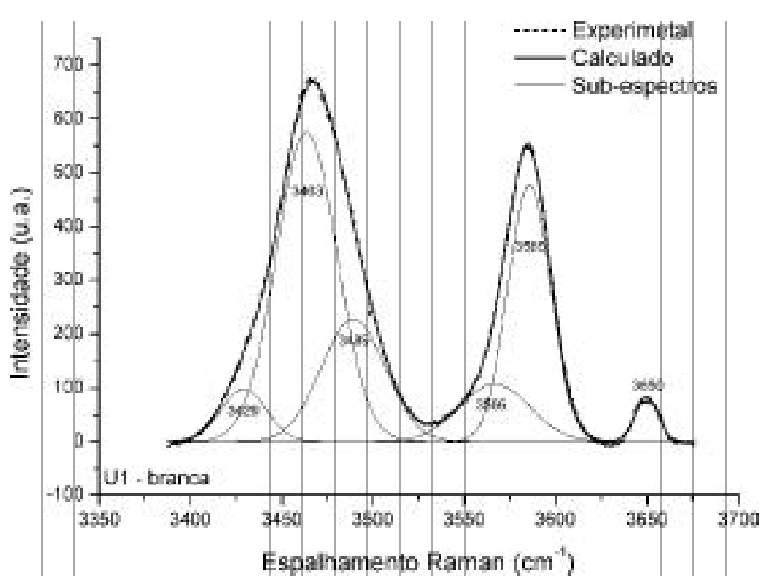

Figura 8 - Espectro Raman na região do estiramento $\mathrm{O}-\mathrm{H}$ de uma elbaíta branca, molécula muito próxima a de um membro final - amostra U1 em polarização $a_{\mathrm{ZZ}}$ (temperatura ambiente).

0,999, sem restrição de ajuste. Para o entendimento dos dados espectrais observados, deve-se, inicialmente, fazer uma tentativa de atribuição das frequiências dos modos vibracionais com a coordenação do ambiente local. Por isso foram utilizadas medidas de luz polarizada em monocristais orientados, para solucionar as dificuldades das atribuições dos espectros. Mesmo assim, a atribuição fica sujeita a alguma incerteza. As bandas do estiramento O-H são observadas com maior intensidade em $\mathrm{a}_{\mathrm{ZZ}}$. Na direção $\mathrm{a}_{\mathrm{ZX}}, \mathrm{a}_{\mathrm{YY}}$ e $\mathrm{a}_{\mathrm{XX}}$ as bandas desaparecem, diagnosticando a direção preferencial paralela ao eixo c.

Corroborando com os dados obtidos do ODEPR, o comportamento espectral das elbaítas é caracterizado por desdobramentos das bandas relativas ao estiramento do $\mathrm{OH}_{3}$, os quais podem estar associados à presença de sítios Y não equivalentes do manganês. 
Esse aspecto diagnostica um certo grau de desordem da estrutura, uma vez que a análise de grupo de fator (Castañeda et al. 2000) mostra que são esperadas apenas duas bandas do estiramento $\mathrm{O}-\mathrm{H}$ do grupo $\mathrm{OH}_{3}$.

\section{DISCUSSÕES E CONCLUSÕES}

Várias atribuições foram feitas para as bandas de absorção óptica dos minerais do grupo da turmalina. Manning $(1969,1973)$ sugere que a banda de absorção a $810 \mathrm{~nm}$ presentes em turmalinas rosa é devida a transições $d$ - $d$ dos íons $\mathrm{Mn}^{3+}$ em sítio octaédrico. De Camargo \& Isotoni (1988) caracterizaram as bandas 788 e $603 \mathrm{~nm}$ como produtos das transições d-d ${ }^{5} \mathrm{E} \rightarrow{ }^{5} \mathrm{~A}$ e ${ }^{5} \mathrm{~B}_{2} \rightarrow{ }^{5} \mathrm{~A}_{1}$ do $\mathrm{Mn}^{3+}$, respectivamente. Henn et al. (1990) acreditam que a cor azul das turmalinas da Paraíba, Brasil, é devida às bandas de absorção com máximos em 700 e $520 \mathrm{~nm}$ causadas por $\mathrm{Cu}^{2+}$ e $\mathrm{Mn}^{3+}$, respectivamente. Reinitz \& Rossman (1988) mostram espectros de elbaítas incolores ricas em $\mathrm{Mn}$ os quais apresentam bandas de absorção fracas a 414 nm na direção de polarização E1c e a 419, 414, 412 e $408 \mathrm{~nm}$ em E//c, causadas por $\mathrm{Mn}^{2+}$ e suas interações com outros constituintes menores. Os espectros de outras amostras incolores ou descoloridas por tratamento térmico mostram as mesmas características em relação ao $\mathrm{Mn}^{2+}$, assim como as $\mathrm{Mn}$ elbaítas de cor amarela esverdeada da Zâmbia, discutidas por Rossman \& Matsson (1986) e Shigley et al. (1984).

As distintas atribuições mostram a complexidade estrutural do grupo da turmalina, o que torna difícil, e quase impossível, fazer atribuições precisas das bandas tendo como base somente os espectros de absorção e a composição química. Vários processos que contribuem diretamente para a causa da cor podem estar envolvidos na origem de tais absorções. Foi feita a integração dos dados da caracterização química, estrutural e óptica das amostras estudadas com a finalidade de atribuir, sem ambigüidade, as bandas de absorção óptica e correlacionar com os centros paramagnéticos geradores de centros cromóforos.

Os processos de excitação que dão origem às bandas do intervalo entre $40.000 \mathrm{a} 4000 \mathrm{~cm}^{-1}$ do espectro eletromagnético podem ser atribuídos a quatro mecanismos: 1) Transferência de carga metal-ligante; 2) Transferência de carga metal-metal; 3) Transições $d$ - $d$ (ou f-f) e; 4) Centros de cor (Langer et al. 1995). As transições eletrônicas entre a divisão dos estados espectroscópicos do campo cristalino de um metal de transição (transições $d$ - $d$ ) causam bandas com intensidades muito maiores que aquelas produzidas pelos outros mecanismos e normalmente apresentamse na faixa do visível, enquanto às de mecanismos de transferência de carga ficam próximas ao ultravioleta (Rossman 1988).

Comparando as bandas dos espectros de absorção óptica $(300 \mathrm{~K})$ no espectro eletromagnético na faixa do visível e das bandas de absorção do dicroísmo circular magnético (1,5 K), obtido do centro paramagnético $\mathrm{Mn}^{2+}$
(ODEPR tagged MCDA), pode-se concluir que, como o único centro paramagnético registrado é do $\mathrm{Mn}^{2+}$, todas as bandas originadas na faixa do visível são devidas a esse íon.

Consistentes com os aspectos estruturais, as duas bandas a 460 e 520nm, que apresentam linhas intermediárias adicionais, finas e juntas, podem ser causadas por duas transições distintas derivadas de ${ }^{2} \mathrm{~T}_{2 \mathrm{~g}} \rightarrow{ }^{2} \mathrm{E}_{\mathrm{g}}$ do ${ }^{[6]} \mathrm{Mn}^{2+}$ localizados em dois sítios $\mathrm{Y}$ magneticamente não equivalentes, respectivamente. Para o $\mathrm{Mn}^{2+}$, em dois sítios Y não equivalentes, era de se esperar dois conjuntos de bandas compostos por, no mínimo, um par de bandas para cada conjunto. Os espectros de absorção óptica, quando ajustados, apresentam duas ou mais bandas, as quais ajustam perfeitamente as bandas centradas a 450 e $520 \mathrm{~nm}$. As freqüências das bandas intermediárias observadas após o ajuste, concordam com aquelas observadas nas transições eletrônicas do estado fundamental para os dois estados excitados do campo cristalino separados pela divisão Jahn-Teller do estado superior ${ }^{2} \mathrm{E}_{\mathrm{g}}$. A banda a $520 \mathrm{~nm}$, pode ser atribuída às transições $d-d$ do $\mathrm{Mn}^{2+}$ em sítio Y(1), mais distorcido, pois origina-se em energias maiores, enquanto a banda a $460 \mathrm{~nm}$ é atribuída às transições $d$ - $d$ do outro $\mathrm{Mn}^{2+}$, localizado em outro sítio $\mathrm{Y}(2)$ menos distorcido.

Por outro lado, a banda mais larga, de menor intensidade e maior energia centrada a $680 \mathrm{~nm}$, pode caracterizar mecanismos de transferência de carga do tipo $\mathrm{Mn}^{2+} \rightarrow \mathrm{Mn}^{3+}$, uma vez que existem dois sítios $\mathrm{Y}$ magneticamente não equivalentes para esse elemento. Transferência de carga metal-metal ou transferência de carga intervalente, causam bandas largas de intensidade menor que as das transições $d$ - $d$ e são fortemente polarizadas ao longo do vetor metal-metal.

O ferro registrado no EPR convencional e nas microanálises não apresenta banda óptica na faixa do visível do MCDA e, portanto, não contribui para a cor rosa. Todas as bandas observadas na faixa do visível no espectro de absorção óptica são similares às apresentadas no MCDA, podendo, dessa forma, serem atribuídas às mesmas transições do manganês. Além disso, o par de bandas $d$ - $d$ pode estar superposto ou submerso no sistema de bandas próximas ao infravermelho e na margem do ultravioleta, respectivamente, como observado no ajuste dos espectros.

Com a integração de todos os resultados espectroscópicos pode-se concluir que a correlação dos centros paramagnéticos $\mathrm{H}^{\circ}, \mathrm{Fe}^{3+}$ e $\mathrm{O}^{-}$com a cor rosa é descartada pelo fato do dicró́smo circular magnético de absorção do espectro de excitação ODEPR ser idêntico ao MCDA total, no qual o único centro paramagnético registrado nesse espectro é do $\mathrm{Mn}^{2+}$. E, finalmente que, o matiz amarelo na cor rosa da amostra MR3 pode ser observado e correlacionado com o centro $\mathrm{Al}-\mathrm{O}^{-}-\mathrm{Al}$, e talvez atribuído à banda de absorção próxima a $630 \mathrm{~nm}$, a qual não está presente no MCDA do espectro de excitação ODEPR. Sabe-se que o centro Al-O- do 
quartzo fumê tem uma banda de absorção larga na região do visível (1,96 e 2,85 eV) e que nos halóides alcalinos as transições de transferência de carga do $\mathrm{H}^{\circ}$ ocorrem no UV em energias acima de $5 \mathrm{eV}$ (Krambrock et al. 2002). Mas isso ainda é um ponto a ser melhor caracterizado em elbaítas que apresentem concentrações maiores desse centro para que a atribuição da banda de absorção óptica seja mais bem examinada.

O grau de ordem-desordem afeta, significativamente, os mecanismos de causa de cor, produzindo centros cromóforos adicionais. As cores rosas das elbaítas são produzidas por teores, significativamente, baixos de $\mathrm{Mn}^{2+}$ distribuídos em dois sítios Y, magneticamente não equivalentes, causando transições $d$ - $d$ distintas produzindo bandas de absorção entre 460 e 520nm. Mecanismos de transferência de carga entre $\mathrm{Mn}^{2+}$ e $\mathrm{Mn}^{3+}$ ocorrem devido à presença de $\mathrm{Mn}^{2+}$ em sítios distintos e estão associados a causas secundárias.

\section{AGRADECIMENTOS}

Este trabalho é parte da Tese de Doutoramento do primeiro autor, que agradece ao CNPq. Os autores são gratos ao Instituto de Geociências da UnB e ao Departamento de Física da UFMG pelo suporte laboratorial.

\section{REFERÊNCIAS}

Bershov, L.V.; Martirossyan, V.O.; Marfunin, A.S.; Plantanov, A.N.; Tarasschan, A.N. 1969. Color centers in lithium tourmaline (elbaite). Soviet Physics Crystallography, 13:629630.

Brodbeck, C.M. \& Bukrey, R.R. 1981. Model calculation for the coordination of $\mathrm{Fe}^{3+}$ and $\mathrm{Mn}^{2+}$ ions in oxide glasses. Physical Review, B24:2334-2342.

Burns, R. G. 1993. Mineralogical application of crystal field theory. Physical and Chemistry of Minerals, 5: 67-88.

Calas, G. 1988. Electron paramagnetic resonance. In Reviews in mineralogy. Spectroscopy methods in mineralogy and geology. V 18, Washington DC, Mineral Soc. Am., 513571.

Castañeda, C., Oliveira, E.F., Gomes, N., \& Pedrosa-Soares, A.C. 2000. Infrared study of $\mathrm{OH}$ sites in tourmaline from the elbaite-schorl series. American Mineralogist, 85: 1503-1507.

De Biasi, R.S. \& Fernandes, A A R. 1984. Measurement of small concentration of $\mathrm{Cr}$ and $\mathrm{Mn}$ in $\mathrm{MgO}$ using EPR. Journal of American Ceramist Soc., 67: C173-C175

De Camargo, M.B. \& Isotani, S. 1988. Optical absorption spectroscopy of natural and irradiated pink tourmaline. American Mineralogist, 73:172-180

Ertl, A.; Hughes, J.M.; Prowatkes, S.; Rossman, G.; London, D. and Fritz, E.A. 2003. Mn-rich tourmaline from Austria: structure, chemistry, optical spectra and relations to synthetic solid solutions.
American Mineralogist, 88:1369-1376.

Gasharova, B.; Mihailova, B.\& Konstantinov, L. 1997. Raman spectra of various types of tourmaline. Physical and Chemistry of Minerals, 4: 935-940.

Hawthorne, F.C. \& Henry, D.J. 1999. Classification of the minerals of the tourmaline group. European Journal of Mineral, 11: 201-215.

Henn, U.; Bank, H.; Bank, F.H. 1990. Transparent bright blue $\mathrm{Cu}$-bearing tourmaline from Paraiba, Brazil. Mineralogical Magazine, 54: 553-557

Holuj, F. \& Manoogian, A . 1968. EPR of $\mathrm{Mn}^{++}$in spodumene. II. Heated crystals. Canadian Journal of Physics, 46: 303-306

Huang, E. 1999. Raman spectroscopy study of 15 gem minerals. Journal of the Geological Society of Chine, 42(2): 301318 .

Kliava, J. 1986. EPR of impurity ions in disordered solids. Physical Status Solidi, B134:411-455.

Krambrock, K.; Pinheiro, M.V.B.; Medeiros, S.M.; Guedes, K.J.; Schweizer, S. \& Spaeth, J.M. 2002. Investigation of radiation-induced yellow color in tourmaline by magnetic resonance. Nuclear Physics (submetido).

Langer, K.; Platanov, N.A.; Rossman, G.R. 1995. Optical Absorption Spectroscopy. In A S. MARFUNIN (ed.) Methods and Instrumentations. Results and Recent Developments. V. 2, Berlin, Springer-Verlag, 109-123.

Lima, R.J.S.; Wegner, R.R. and Suassuna, J.F. 2003. Color center and dichroism investigation in some Brasulian species of tourmaline using optoacoustic tecnique. Annais of Optics, XXVI ENFM, V5.

Manning, P.G. 1969. Optical absorption spectra of chromium bearing tourmalines, black tourmaline and buerguerite. Canadian Mineralogist, 10:57-70.

Manning, P.G. 1973. Effect of second-nearest-neighbor interaction on $\mathrm{Mn}^{3+}$ absorption in pink and black tourmalines. Canadian Mineralogist, 11:971-977.

Mcgavin, D.G.; PALMER, R.A ; TENNANT, W.C.\& DEVINE, S.D. 1982. Use of ultrasonically modulated electron resonance to study S-state ions in mineral crystal: $\mathrm{Mn}^{2+}$ and $\mathrm{Fe}^{3+}$ in tremolite. Physics and Chemistry of Minerals, 8: 200-205.

Oliveira, E.F.; Castañeda, C.; Eeckhout, S.G.; Gilmar, M.M.; Kwitko, R.R; De Grave, E, \& Botelho, N.F. 2002. Infrared and Mössbauer study of Brazilian tourmalines from different geological environments. American Mineralogist, (submetido e aceito)

Pinheiro, M.V.B.; Lameiras, F.S.; Krambrock, K.; Karfunkel, J. \& Silva, J.B. 1999. Irradiated quartz: electron spin resonance study. Australian Gemmology, 207: 285.

Reinitz, I.M. \& Rossman, G.R. 1988. Role of natural radiation in tourmaline coloration. American Mineralogist., 73: 822825 .

Rossman, G.R. \& Mattson, S.M. 1986. Yellow, Mn-rich elbaite with Mn-Ti intervalence charge transfer. American Mineralogist, 71 (3/4): 599 - 602.

Rossman, G.R. 1988. Optical Spectroscopy. In: F.C. Hawthorne (ed) Spectroscopic methods in mineralogy and geology, Ver. Mineral, 18: 207-254.

Shaffer, J.S; Farach, H.A \& Poole, C.P. 1976. Electron spin resonance study of manganese-doped spinel. Physics Review, B13: $1869-1875$.

Shigley, J.E., Stockton, C.M. 1984. "Cobalt-blue" gem spinels. Gems \& Gemology, 20(1): 34-41. 